\title{
GRAVITATIONAL LENSING IN LOW-REDSHIFT CLUSTERS OF GALAXIES: THE ARC-LIKE OBJECT IN ABELL 3408 AND ITS LENSING INTERPRETATION
}

\author{
Luis E. Campusano ${ }^{1}$ \\ Observatorio Astronómico Cerro Calán, Departamento de Astronomía, Universidad de Chile, Casilla 36-D, Santiago, Chile \\ JEAN-PAUL KNEIB \\ Observatoire Midi-Pyrénées, 14 Av. E.Belin, 31400 Toulouse, France \\ EDUARDO HARDY \\ Département de Physique, Observatoire du mont Mégantic, Université Laval, Sainte-Foy, P.Q., Canada, G1K 7P4 \\ Accepeted to The Astrophysical Journal (Letters)
}

\begin{abstract}
We analyze the seldomly discussed lensing effects expected in low-z clusters $(\mathrm{z}=0.05-0.15)$, using as an example the bright arc $(z=0.073)$ discovered by Campusano and Hardy(1996) near the central elliptical galaxy of the cluster Abell $3408(z=0.042)$. The photometric and spectroscopic observations for both the central elliptical and the arc are presented. The mass distribution in A3408 is modeled by scaled versions of two representative distributions derived from studies of clusters at higher redshifts: i) a "minimum" mass case where the mass distribution follows the light profile of the central elliptical galaxy and, ii) a "maximum" mass case where a typical massive dark halo is added to the previous case. The observed arc is well reproduced by both models, but rather small magnifications of the source galaxy are implied. The source galaxy is tentatively identified in both the lensing and non-lensing scenarios as being a spiral. The smaller lensed spiral $\left(14.6 h_{50}^{-1} \mathrm{kpc}, M_{B}=-18.2\right)$ predicted by the dark halo model appears to fit the observations marginally better. Furthermore, we found that only the dark halo model predicts a measurable amount of weak shear in the images of faint background galaxies. We conclude that observations, under very good seeing conditions, of week shear in faint background galaxies in the direction of low-redshift galaxy clusters are possible. The combination of the latter with X-ray data, can provide a powerful tool to probe the mass distribution in the very central region of galaxy clusters.

Subject headings: galaxies:clusters:general — gravitational lensing — galaxies:clusters: individual(Abell 3408)
\end{abstract}

\section{INTRODUCTION}

Clusters of galaxies are complex gravitationally bound systems holding together three components: galaxies, ranging from the faintest irregulars to the brightest elliptical galaxy that usually sits at the center of the potential well, hot gas [HG] (detected by its X-ray emission) and dark matter $[\mathrm{DM}]$. Many recent studies of the mass distribution of galaxy clusters exist, probing the distribution with various tools: galaxy dynamics (e.g. the CNOC survey, Carlberg et al 1997), X-ray analysis (e.g. Allen, Fabian \& Kneib 1996, Pislar et al 1997) and gravitational lensing (e.g. Fort \& Mellier 1994, Narayan \& Bartelmann 1995). Although, they are all generally consistent with a density profile that goes like $\rho \sim 1 / r^{2}$ at intermediate scales (200-1000 kpc), the exact profile at small and large scales is poorly known. At small radii there is usually a large number of galaxies in the central part of the clusters, being difficult to disentagle the galaxy halo masses from the larger-scale cluster component. At large radii the data are too noisy to make any safe statements. Numerical simulations by Navarro, Frenk \& White $(1995,1996)$ indicate that simple gravitational systems follow a 'Universal' mass profile with a $\rho \sim 1 / r$ cusp at the center. However this results is subject to debate and other groups have claimed a steeper or shallower central profile (Kravstov et al 1997, Moore et al 1997). Generally, due to the presence of the brightest central galaxy the total mass distribution will be cuspy (on a scale of a few $\mathrm{kpc}$ ) since elliptical galaxies are well fitted by a de Vaucouleurs profile. Does the cusp exist only on the stellar component, or is it also a property of the HG and DM components? Solving these questions will help understand what the dynamical interactions between the various components are, and whether any simple scaling laws can be found.

The investigation of gravitational lensing of faint background galaxies by low-redshift clusters of galaxies has been largely unexplored so far. In fact, background galaxies offer the possibility of probing the mass distribution of these clusters with higher-spatial resolution than that allowed by high- $z$ cluster lenses, and should yield more accurate results on scales appropriate to the study of the mass profile of the cluster core or even of individual galaxy members. The study of low- $z$ cluster lenses has specially good prospect now that efficient wide-field cameras will soon become available (e.g. the CFHT12K and the ACS aboard HST).

Theoretically, the cluster lensing efficiency —assuming a non-evolving mass profile with redshift, and a reasonable redshift distribution for the faint galaxy population (see for example Natarajan \& Kneib 1996) - has its maximum around $z \sim 0.2$. Thus, a cluster at $z \sim 0.1$ is as efficient, in terms of the mean shear produced at a cer-

\footnotetext{
${ }^{1}$ Visiting astronomer, Cerro Tololo Inter-American Observatory, National Optical Astronomy Observatories, which are operated by the Association of Universities for Research in Astronomy, Inc. under contract with the National Science Foundation.
} 
tain angular distance from its center, as a $z \sim 0.3$ cluster. For low redshift clusters the lensing efficiency depends on the total mass profile at the center, whereas for high redshift clusters it depends on the redhift distribution of the background faint galaxies.

There is already evidence of lensing in low redshift clusters of galaxies, but a discussion of the use of these cluster lenses for the investigation of the mass profile of clusters has not yet been addressed. Allen, Fabian \& Kneib (1996) discovered a $z=0.43$ arc in the PKS0745 $z=0.10$ cluster, which has been successfully modelled as a gravitational lens image. A second case has been suggested by Shaya, Baum and Hammergren (1996), who found an arclike feature- so far without redshift information - close to NGC4881 ( $z=0.024)$ in the Coma cluster. And thirdly, there is the tentative case found by Campusano \& Hardy (1996) of a bright $z=0.073$ arc-like feature in a $z=0.042$ cluster (Abell 3408). In this paper, we further investigate the lensing by low- $z$ clusters by discussing the possibility that the arc in Abell 3408 is a gravitational image, and by computing the elliptical distorsions expected in the images of the faint background galaxies (weak shear) under different mass models. We adopt $\Omega_{0}=1, \lambda=0$ and $\mathrm{H}_{0}=50$ $h_{50}^{-1} \mathrm{~km} \mathrm{~s}^{-1} \mathrm{Mpc}^{-1} ; 1$ " corresponds to $1.136 h_{50}^{-1} \mathrm{kpc}$ at the distance of the cluster. 


\section{OBSERVATIONS}

Little is known about A3408 $(z=0.042)$, whose J2000.0 coordinates are $\mathrm{RA}=07^{h} 08^{m} 29^{s}, \mathrm{DEC}=-49^{\circ} 12^{\prime} 50.3^{\prime \prime}$. It lies in a largely unexplored low galactic latitude section of the southern sky and it may even form a single unit with A3407 (Galli et al. 1993). Abell 3408 has been detected by the Rosat-All-Sky-Survey which measured a flux $L_{X}=0.5 \times 10^{44} \mathrm{erg}^{-1}$ (Ebeling et al 1996). Recently, Campusano and Hardy(1996) discovered an arc-like feature $(z=0.073)$ near its center. We present here - in full - the photometric and spectroscopic observations of the arc and the central elliptical galaxy of A3408.

\subsection{Photometry}

Imaging observations trough the $\mathrm{B}, \mathrm{R}$ and I filters were obtained with the $\mathrm{CF} / \mathrm{CCD}$ camera system at the Cerro Tololo Inter-American Observatory (CTIO) 0.9-m telescope. The detector was the $2048 \times 2048$ Tek $2 \mathrm{k} \# 3 \mathrm{CCD}$, which has a scale of 0.40 "/pixel. Landolt(1992) standard stars observation were used for the photometric calibration. The discovery image was obtained serendipitously during observations of southern galaxy clusters as part of a project conducted in collaboration with Dale, Giovanelli, \& Haynes (Cornell).

Fig. 1 displays the combination of the $\mathrm{B}$ and I images of the A3408 cluster. The field size is $6^{\prime} \times 8^{\prime}$ and is centered on the brightest elliptical of the cluster. An inspection of Fig. 1 reveals an arc-like object $\sim 50$ " away from the brightest central elliptical galaxy; its orientation suggesting the possibility of a gravitational lensing effect. Fig. 3 is a zooming of the R-band image showing the arc and the central galaxy. The width of arc-like object is unresolved, with a FWHM of $\sim 1.2$ " compatible with the observed seeing. Its magnitude and color are (after removal of the contiguous stellar object from the images via DAOPHOT) as follows : $R=18.60, R-I=0.42$, and $B-R=0.79$. The mean surface brightness values are $\mu_{B}$ $=24.23 \mathrm{mag} / \operatorname{arcsec}^{2}, \mu_{R}=23.44 \mathrm{mag} / \operatorname{arcsec}^{2}$, and $\mu_{I}=$ $23.02 \mathrm{mag} / \mathrm{arcsec}^{2}$. The length of the arc is $\sim 11 "$.

Using the ellipse package in IRAF/STSDAS we computed the surface photometry of the central elliptical in $\mathrm{R}$ and I band. The surface brightness profile is well fitted by a de Vaucouleurs profile with effective radius $\mathrm{R}_{e}=19.3 \pm 3$ $\operatorname{arcsec}\left(22 \pm 4 h_{50}^{-1} \mathrm{kpc}\right)$ and $\mu_{E}(I)=21.5 \mathrm{mag} / \operatorname{arcsec}^{2}$, $\mu_{E}(R)=22.3 \mathrm{mag} / \operatorname{arcsec}^{2}$. This galaxy has a small ellipticity $\varepsilon=\left(a^{2}-b^{2}\right) /\left(a^{2}+b^{2}\right)=0.08$ and an orientation of $35^{\circ} \pm 5^{\circ}$. Its total $\mathrm{B}$ magnitude is 15.0 or $\mathrm{M}_{B}=-22.0$ $\left(\mathrm{L}_{B}=1.0 \times 10^{11} \mathrm{~L}_{B \odot}\right)$.

\subsection{Spectroscopy}

Spectroscopic observations were carried out with the Ritchey Chretien spectrograph at CTIO $4 \mathrm{~m}$ telescope. The detector was the Loral 3k CCD, which after binning in the dispersion direction generated a readout format of 1595 pix $\times 785$ pix. We used a 350 lines $\mathrm{mm}^{-1}$ grating blazed at $4400 \AA$ giving a wavelength coverage of $\sim 3200-8800 \AA$ and a spectral resolution of $\sim 8 \AA(1.5$ " slit). The position of the slit along the arc approached the parallactic angle. Flux calibration was determined by observations of the southern spectroscopic standards of Hamuy et al. (1994).
We obtained absorption spectra for the central elliptical galaxy (yielding $z=0.0419 \pm 0.0020$, in good agreement with the results of Galli et al. 1993), and for the stellar object inmediately next to the arc, which was found to be a a K-type foreground star.

For the arc, its relatively high surface brightness yielded a high S/N integrated spectrum (Fig. 2). Its derived mean redshift, based on the eight well-measured nebular emission lines (listed below), is $z=0.0728 \pm 0.0010$ corresponding to $21,840 \pm 30 \mathrm{~km} \mathrm{~s}^{-1}$. We also detected the absorption A-type spectrum of the underlying stellar population of the source which confirmed the emission-line velocity from six absorption lines. Also, using the most prominent nebular lines ( [OII] $3727 \AA$, [OIII] $5007 \AA$ and $\mathrm{H} \alpha$ ) we measured velocities along the arc, which revealed a velocity gradient over a linear distance of about $10 \mathrm{kpc} h_{50}^{-1}$ with $\sim 80 \mathrm{~km} \mathrm{~s}^{-1}$ peak-to-peak amplitude.

Equivalent widths (in $\AA$, indicated within parenthesis) for the main nebular lines are as follows: [OII] $\lambda 3727$ (24.5); $\mathrm{H} \beta$ (6.7); [OIII] $\lambda 4959$ (8.7); [OIII] $\lambda 5007$ (20.0); $\mathrm{H} \alpha$ (58.7); [NII] $\lambda 6583$ (5.0); [SII] $\lambda 6717$ (12.1). We are clearly not seeing a young starburst as the Balmer lines are not particularly broad $-\mathrm{H} \alpha$ and $\mathrm{H} \beta$ both are well fitted by gaussians with a FWHM of $8 \AA$ (i.e., $310 \mathrm{~km} \mathrm{~s}^{-1}$ ), equivalent to the instrumental resolution-, and the $\mathrm{EW}$ value of $6.7 \AA$ for $\mathrm{H} \beta$ indicates an old age for the starforming region from the models of Leitherer \& Heckman (1995). The overall emission spectrum is characteristic of intermediate-excitation HII regions with a ratio [OIII $] \lambda$ $5007 / \mathrm{H} \beta=3.0$, and low ratio $[\mathrm{NII}] / \mathrm{H} \alpha=0.09$. The underlying stellar spectrum with well-defined Balmer lines is compatible with an intermediate-A average spectral type. Thus, the spectral information is suggestive of a late-type spiral, an Irregular, or a blue compact dwarf [BCD] galaxy.

The linear size of the arc is $20.6 h_{50}^{-1} \mathrm{kpc}$, a value more compatible with a medium-size spiral than with a typical Irr or BCD, but its rotational velocity is about half of that expected for an edge-on spiral of this luminosity (Mathewson \& Ford. 1996). Notice also, that the implied absolute magnitude of the arc-like object is $M_{B}=-18.8$, which is too faint for a medium-size spiral but not incompatible with that of a small spiral such as M33, or an irregular such as the LMC, both of which however have small linear sizes of only a few (6-7) kpc. Also, the mean surface brightness of the arc is too low for a medium-size spiral, but again compatible with a small spiral, an irregular, or a BCD.

Therefore, the straightforward interpretation of the arc implies a physical object of somewhat contradictory properties. It appears to be an elongated extragalactic object with emission characteristic of a typical HII region along most of its body, $20.6 h_{50}^{-1} \mathrm{kpc}$ in size, a velocity profile with amplitude $\sim 40 \mathrm{~km} \mathrm{~s}^{-1}$, and a surface brightness and luminosity typical of the much smaller (factor 4) Magellanic irregular galaxies. A low luminosity can hardly be attributed to dust as the colors are normal. But, could this arc-like object be the product of gravitational lensing by the angularly close foreground giant elliptical? 


\section{GRAVITATIONAL LENSING IN ABELL 3408}

There is no evidence for a counter image of the arc- although there are objects of similar color to the arc, they are not at the position expected for a "two-image" configuration. The "gravitational radius" of the arc is $\sim 50^{\prime \prime}$ (i.e. $\sim 56.8 h_{50}^{-1} \mathrm{kpc}$ at the cluster distance).

For a study of the gravitational lens scenario, we used both a simple spherical and a more sophisticated elliptical lensing models in order to compute the projected mass of the cluster within the radius defined by the bright arc. All these potentials are able to produce a single arc, as observed, but each one with different implications for the source galaxy and the physical parameters of the cluster. We consider below two lensing mass model to compute the source properties (see Table 1) of the "single" arc:

i) The constant $M / L$ model: Given that the arc is at a distance of $\sim 3 \mathrm{R}_{e}$, we adopted a mass distribution that follows the light distribution - assuming a constant $\mathrm{M} / \mathrm{L}_{B} \sim 10$ which is consistent with the results from the fundamental plane -, and which can be regarded as the "minimum" mass case. The mass enclosed within $R_{\text {arc }}$ is $\mathrm{M}\left(R_{\text {arc }}\right) \sim 1.5 \times 10^{12} \mathrm{M}_{\odot}$, and the velocity dispersion of the stars at $\mathrm{R}_{e} / 2$ is $225 \mathrm{~km} \mathrm{~s}^{-1}$. The model predicts an amplification of $0.01 \mathrm{mag}$ and a tangential stretching of $\sim 1.02$ - there is obviously no much difference with respect to the no-lensing case. The predicted weak-shear $\gamma$ at the distance of the arc, for galaxies with $<z>\sim 0.7$, is $\gamma \sim<\varepsilon_{I}>\sim 0.07\left(<\epsilon_{I}>\right.$ is the expected averaged mean ellipticity of the imaged faint galaxies - see e.g. Kneib et al 1996). This value is at the detection limit.

ii) The dark halo model: Both, strong and weak lensing studies (e.g. Kneib et al 1996) clearly indicate the presence of dark halos in clusters, centered on the brightest elliptical galaxy, and with a typical $\mathrm{M} / \mathrm{L}_{V}$ ratio of $\sim 200$. Such dark haloes are also supported by the high velocity dispersion of cluster galaxies, as well as by the increase of the velocity dispersion of stars in giant elliptical galaxies (e.g. A2029, Dressler 1980). Adopting a massive mass profile comparable to the one found in A2218 (Kneib et al $1996)$ - the "maximum" mass model - we predict a single observed arc with an enclosed mass of $\mathrm{M}\left(R_{\text {arc }}\right) \sim 4.4 \times 10^{13}$ $\mathrm{M}_{\odot}$ corresponding to $M / L_{B}\left(R_{\text {arc }}\right) \sim 230$, and an asymptotic velocity dispersion for the member galaxies of 900 $\mathrm{km} s^{-1}$. The model predicts an amplification of $0.6 \mathrm{mag}$ and a tangential stretching of $\sim 1.4$ for the source galaxy.

In contrast to the prediction of the 'minimum' mass model, the expected weak-shear at the distance of the arc, for galaxies with $\left\langle z>\sim 0.7\right.$, is $\left\langle\varepsilon_{I}>\sim 0.5\right.$. This value is quite large and readily measurable, thus it is a strong prediction of this model.

The velocity dispersion of the stars in the central elliptical galaxy is predicted to vary from $275 \mathrm{~km} \mathrm{~s}^{-1}$ at the center, for both the low-mass and dark-halo models, to $225 \mathrm{~km} \mathrm{~s}^{-1}$ and $435 \mathrm{~km} \mathrm{~s}^{-1}$ at $\mathrm{R}_{e} / 2$, and to 190 and 550 $\mathrm{km} s^{-1}$ at $\mathrm{R}_{e}=1$, for model i) and ii) respectively.

Note that this model should be considered as a fiducial one, describing the mass distribution of A3408 only in an approximate manner. In this exploratory work, the idea was simply to extrapolate the mass distribution from a higher- $z$ cluster and scale it to a lower redshift. The possibility of adopting a more massive halo model, in or- der to get a better agreement between the source galaxy a BCD, was considered and discarded. A much larger total mass was required, which would have placed this cluster amongst the most massive ones in the Universe in spite of its relatively low X-ray luminosity. 


\section{DISCUSSION AND CONCLUSION}

But how common are the derived 'source' galaxy characteristics? We are facing two possibilities: a) an - essentially - unlensed elongated galaxy of $20.6 h_{50}^{-1} \mathrm{kpc}$ and $M_{B}=-18.8$, or b) a lensed elongated source of $14.6 h_{50}^{-1}$ $\mathrm{kpc}$ and $M_{B}=-18.2$. We can derive a-priori probabilities from an examination of the Binggeli, Sandage \& Tamman (1988; Fig. 1) luminosity functions which also assume $\mathrm{H}_{0}=$ $50 \mathrm{~km} \mathrm{~s}^{-1} \mathrm{Mpc}^{-1}$.

For case a) $35 \%$ of the field galaxies will be Sc's with the required absolute magnitude, but for the observed edge-on configuration only $\sim 5-10 \%$ are possible, and the probability drops significantly when the low mass implied by the low rotation observed is considered. Even the smallest Sc's have twice the observed rotational velocity. For later spiral types $(\mathrm{Sd}+\mathrm{Sm})$ the corresponding probabilities are about $50 \%$ lower. Irregulars or BCD's with this absolute magnitude are essentially non-existent. For case b) the situation does not change significantly due to the shallow slope of the observed LF: somewhat less than $30 \%$ of the field galaxies will be Sc's with the required magnitude, but for the observed edge-on configuration only $\sim 5 \%$ are possible; in this case, the low mass implied by the low rotation observed is perhaps a bit more realistic. At this absolute magnitude Irr and BCDs still remain virtually nonexistent. Both the mean surface $\mu_{B}=24.23 \mathrm{mag} / \mathrm{arcsec}^{2}$ - although typical of the Magellanic irregular galaxies and color B-R $=0.79$ of the arc are not incompatible with those of small Sc galaxies. Then, a small lensed spiral seen nearly face on, and thus of low projected rotational velocity, but elongated by gravitational shear would fit the observations.

The above probabilities, however, are strictly a-priori and thus must be taken with a grain of salt as we cannot at present exclude that the observed arc is not simply a conspiring and unusual unlensed member of the astronomical zoo. We cannot at this point decide on the identity of the source, but on statistical grounds a spiral seems likely, although we cannot decide either on whether such a spiral would or not be lensed, and if it is, what its intrinsic orientation would be.

On a more positive note, however, we have shown that there is a strong test that can be applied to the dark halo model: deep wide-field imaging of low- $z$ clusters (similar to A3408) should display a weak shear easily measurable with standard techniques. The analysis of such weak shear, coupled with X-ray observations of these clusters, should unveil the profile of the different mass components in the very central region of the cluster of galaxies. A better understanding of the dynamics of these relaxed systems would then be possible.

This research was supported by ECOS-CONICYT grant C95U02, by FONDECYT grant No. 1970735 to LEC, by CNRS to JPK, and by NSERC-Canada and FCARQuébec to EH. We acknowledge the excellent technical support of the CTIO staff and thank Guy Mathez for his encouragement. 


\section{REFERENCES}

Allen, S.W., Fabian, A.C. \& Kneib, J.P., 1996, MNRAS 279, 615

Binggeli, B., Sandage, A., R., \& Tammann, G. ,A., 1988, ARA\&A 26,509

Campusano, L.E. \& Hardy, E., 1996, in Astrophysical Applications of Gravitational Lensing, p.125, Kluwer (eds. Kochanek,C.S. and Hewitt,J.N.)

Carlberg, R.G. et al., 1997, ApJ, 476, L7

Dressler, A., 1981, ApJ 243, 26

Ebeling, H., Voges, W., Böhringer, Edge, A.C., Huchra, J.P. and Briel, U.G., 1996, MNRAS, 281, 799

Fort, B., Mellier, Y., 1994, Astron. Astr. Rev., 5, 239

Galli, M., Cappi, A., Focardi, P., Gregorini, L. \& Vetolani, G., 1993, A\&AS, 101, 259

Hamuy, M. et al., 1994, PASP, 106, 566

Heckman, Armus \& Miley 1987, AJ, 93, 276

Kneib, J-P., Ellis, R.S., Smail, I., Couch, W. \& Sharples, R.M., 1996, ApJ, 471, 643

Kravtsov, A.V., Klypin, A.A., Bullock, J.S., Primack, J.R., 1997,
ApJ submitted, astro-ph/9708176.

Leitherer, C., \& Heckman, T. M., 1995, ApJS, 96, 9

Mathewson, D. S., \& Ford, V. L., 1996, ApJS, 107, 97

Narayan, R. \& Bartelmann, M., 1995, in Formation of Structure in the Universe, Proc. 1995 Jerusalem Winter School, in press (eds. Dekel, A. \& Ostriker, J.P.)

Natarajan, P. \& Kneib, J-P., 1997, MNRAS, 287, 833

Navarro, J.F., Frenk, C.S. \& White, S.D.M., 1995, MNRAS, 275, 720

Navarro, J.F., Frenk, C.S. \& White, S.D.M., 1996, ApJ, 462, 563

Moore, B., Governato F Quing, T., Stadel, J., Lake, G., 1997, ApJL submitted, astro-ph/9709051

Pislar, V., Durret, F., Gerbal, D., Lima Neto, G. B., Slezak, E., 1997, A\&A 322, 53

Shaya, E.J., Baum, W.A. \& Hammergren, M., 1996, in Clusters, Lensing and the Future of the Universe, ASP Conf Ser, 88, p.237 (eds. Trimble, V. \& Reisenegger, A.) 


\begin{tabular}{llllll}
\hline Model & $\begin{array}{l}\mathrm{R}_{\text {intr }} \\
(\mathrm{mag})\end{array}$ & $\begin{array}{l}\Delta m \\
(\mathrm{mag})\end{array}$ & $\begin{array}{l}\text { Length } \\
(\operatorname{arcsec} / \mathrm{kpc})\end{array}$ & $\begin{array}{l}\mathrm{M}_{B} \\
(\mathrm{mag})\end{array}$ & Comments \\
\hline Unlensed & 18.6 & 0 & $11.0 / 20.6$ & -18.8 & \\
Constant M/L & 18.6 & 0.01 & $10.8 / 20.3$ & -18.8 & $\mathrm{M} / \mathrm{L}_{B}=10$ and $<\varepsilon_{I}>\sim 0.07$. \\
Dark halo & 19.2 & 0.60 & $7.8 / 14.6$ & -18.2 & $\mathrm{M} / \mathrm{L}_{B} \sim 230$ and $<\varepsilon_{I}>\sim 0.50$. \\
\hline
\end{tabular}

Table 1: The implied intrinsic properties of the arc under three hypotheses: no-lensing and lensing under two mass profiles. The predicted mean ellipticity of the faint background galaxies $\left.\left(<\varepsilon_{I}\right\rangle\right)$ and the Mass/Luminosity ratio, both evaluated at the distance of the arc from the giant elliptical galaxy, are given for the lensing models.

Fig. 1. $-6^{\prime} \times 8^{\prime}$ field showing the central region of the A3408 cluster. An arc-like object is seen 50" away from the brightest central elliptical galaxy. The pseudo-colors, obtained from B and I CCD frames taken with the 0.9-m CTIO telescope, were adjusted so that they approximately match the real color contrast of the objects. North is at the left and East at the bottom. 


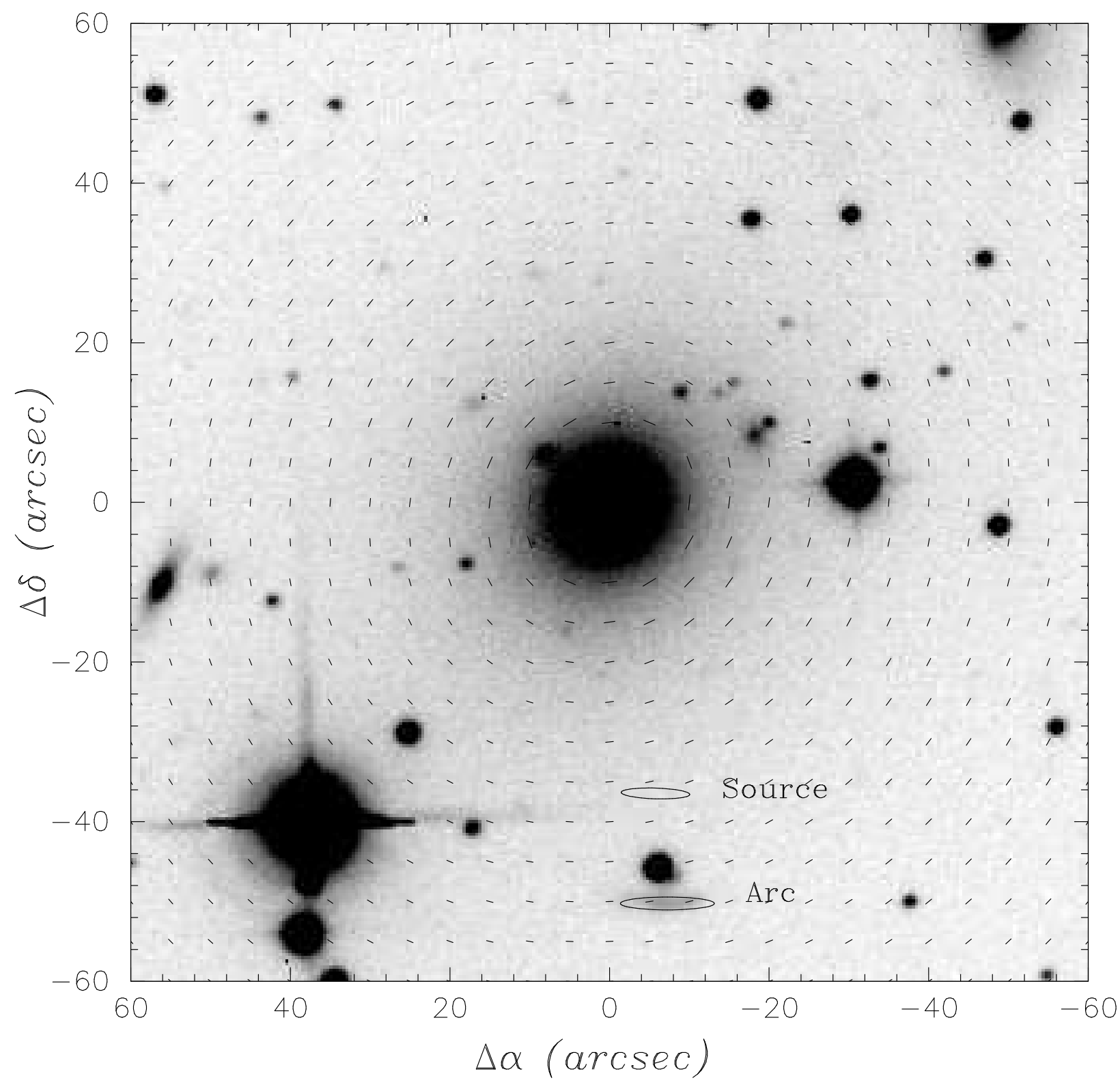

Fig. 3.- Dark halo model. Zooming of R-band image taken with the 0.9-m CTIO telescope. The observed arc is at the bottom, while the derived position of the source is marked with an ellipse. The predicted shear field, for background galaxies with a mean redshift of $\sim 0.7$, is overlaid; it ranges from $\left\langle\varepsilon_{I}\right\rangle \sim 0.3$ in the corners to $\left\langle\varepsilon_{I}\right\rangle \sim 0.7$ in the central part. North is at the top and East at the left. 
This figure "a3408ib.jpeg" is available in "jpeg" format from: http://arxiv.org/ps/astro-ph/9712069v2 